\title{
Joint accurate time and stable frequency distribution infrastructure sharing fiber footprint with research network
}

\author{
Josef Vojtech, ${ }^{\mathrm{a}, \star}$ Martin Slapak, ${ }^{\mathrm{a}}$ Pavel Skoda, ${ }^{\mathrm{a}}$ Jan Radil, ${ }^{\mathrm{a}}$ Ondrej Havlis, ${ }^{\mathrm{a}}$ Michal Altmann, ${ }^{\mathrm{a}}$ Petr Munster, ${ }^{\mathrm{a}}$ Radek Velc, ${ }^{\mathrm{a}}$ \\ Jan Kundrat, ${ }^{a}$ Lada Altmannova, ${ }^{a}$ Rudolf Vohnout, ${ }^{a}$ Tomas Horvath, ${ }^{a}$ Miloslav Hula, ${ }^{a}$ Vladimir Smotlacha, ${ }^{b}$ Martin Cizek, \\ Lenka Pravdova, ${ }^{c}$ Simon Rerucha, ${ }^{c}$ Jan Hrabina, ${ }^{c}$ and Ondrej Cip ${ }^{c}$ \\ ${ }^{a}$ CESNET, a.l.e., Department of Optical Networks, Prague, Czech Republic \\ ${ }^{\mathrm{b}}$ CESNET, a.I.e., Department of Technologies for Network Applications, Prague, Czech Republic \\ Institute of Scientific Instruments of the CAS, Department of Coherent Optics, Brno, Czech Republic
}

\begin{abstract}
The infrastructure essentialities for accurate time and stable frequency distribution are presented. Our solution is based on sharing fibers for a research and educational network carrying live data traffic with time and frequency transfer in parallel. Accurate time and stable frequency transmission uses mainly dark channels amplified by dedicated bidirectional amplifiers with the same propagation path for both directions of transmission. This paper targets challenges related to bidirectional transmission, particularly, directional nonreciprocities. $\odot$ The Authors. Published by SPIE under a Creative Commons Attribution 3.0 Unported License. Distribution or reproduction of this work in whole or in part requires full attribution of the original publication, including its DOI. [DOI: 10.1117/1.OE.56.2.027101]
\end{abstract}

Keywords: accurate time; stable frequency; wavelength division multiplexing; bidirectional reciprocal path; Sagnac effect.

Paper 161695P received Oct. 28, 2016; accepted for publication Jan. 12, 2017; published online Feb. 6, 2017.

\section{Introduction}

Accurate time and stable frequency helps to improve the accuracy and resolution of a wide range of precise measurements; it also represents essential prerequisites for a broad range of fields, e.g., different flavors of sensing, Earth sciences, metrology, navigation, geodesy, astronomy, radioastronomy, seismology, fundamental physics, etc. The use of an optical carrier with a frequency of hundreds of $\mathrm{THz}$ outperforms traditional radio frequency-based methods relying on much lower frequencies. ${ }^{1}$ Essentially, the length of a propagation path through terrestrial infrastructures is shorter, and the path itself can be greatly stabilized compared to satellite-based methods. In general, the terrestrial optical time and frequency (TF) transfer has been facing increased interest in recent years, e.g., Refs. 2-10.

Previous works have dealt with free-space optical transmission methods, e.g., Ref. 1, and their approach faced the turbulent behavior of the Earth's atmosphere. Recent works aiming to overcome large distances are oriented toward fiber transmission, where a number of projects are using dedicated fibers ${ }^{2,3}$ or fibers shared with data transmissions. ${ }^{4,6-10}$ A stable propagation delay in fiber is a crucial parameter for stable and accurate transmissions, but, in addition, optical fibers are influenced by environmental changes around the optical infrastructure. These include not only temperature changes but also mechanical disturbance and acoustic vibrations. Different compensation techniques have been developed, and the majority of them are based on the reciprocity of transport delay between locations $\mathrm{A}$ and $\mathrm{B}: \delta=\delta \mathrm{AB}=\delta \mathrm{BA}$. To achieve such reciprocity in transmission requires utilizing single fiber bidirectionally, so slow acting disturbances will cancel in the first order. This is a very challenging task

*Address all correspondence to: Josef Vojtech, E-mail: josef.vojtech@cesnet .cz over long distances, especially when bidirectional optical amplification needs to be deployed. The use of bidirectional amplifiers is limited, namely due to reflections and backscattering from the real optical line. However, methods using a standard fiber pair have been developed and realized; the details can be found in Refs. 4, 8, and 9. Practical implementation of single-fiber bidirectional transmission requires utilization of some kind of directional multiplexing; otherwise, it is impossible to distinguish a useful signal from a spurious one (amplified back reflections and backscattering). In most cases, a wavelength division multiplexing is used, but occasionally, a time division multiplexing can be found. ${ }^{5}$

The paper is structured as follows. Section 1 introduces the paper. Section 2 introduces the TF infrastructure under development, the concepts used for spectral sharing between transmissions, and the method for frequency transfer stabilization. Section 3.1 discusses directional nonreciprocities caused by relativistic effects and shows the results for the major links of the TF infrastructure, Section 3.2 discusses directional nonreciprocity caused by a wavelength walk-off of transmitters and shows the concept allowing a more precise determination of this value in a real network environment. Finally, Section 4 concludes the paper.

\section{Infrastructure}

The CESNET association (the e-Infrastructure for research and education provider in the Czech Republic) began development of $\mathrm{TF}$ infrastructure in $2011 .^{8,11}$ The total line length of TF transmissions within the CESNET's network is about $1683 \mathrm{~km}$ and roughly $67 \%$ of the infrastructure length $(1137 \mathrm{~km})$ is in operation, as is shown in Fig. 1. The shared model of joint TF and data has been chosen because of economic reasons, high costs for fiber leasing. The infrastructure allows the parallel transmission of accurate time and stable frequency distribution, based on wavelength 


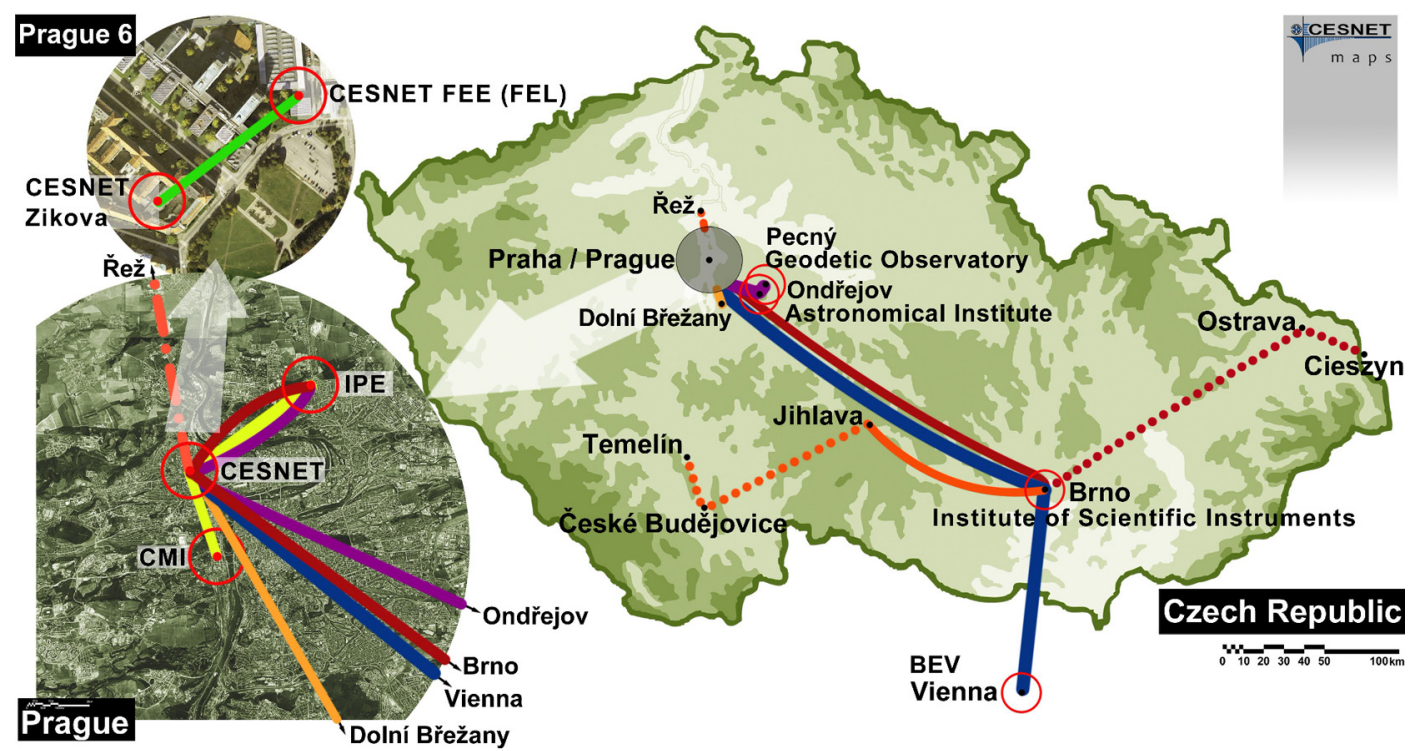

Fig. 1 TF infrastructure.

division multiplexing (WDM). Another project introduced the feature of a joint TF transmission, ${ }^{6}$ however, using T modulated into a frequency signal, which can be quite nonversatile if only a $\mathrm{T}$ transmission is required. Recently, the WDM approach has been introduced further by the team from Ref. 10. The two-way time transfer is based on proprietary time transfer adapters (TTAs), which have been designed using a field programmable gate array; for more details, see Ref. 8. TTAs are capable of operating in heterogeneous environments, including multimode fibers, singlemode dedicated fibers, telecom lambdas, and passive channels within other WDM systems. Also, the transmission is performed over a single fiber bidirectional channel and traditional fiber pair setups. More details can be found in Ref. 12 .

The WDM approach mentioned above is also often used for directional multiplexing to distinguish a useful signal from spurious reflections and cross-talks. The same applies for the presented infrastructure. Time transmission utilizes the standard ITU-T grid, and the spectral walk-off was set to $100 \mathrm{GHz}$ due to poor commercial availability of singlechannel filters for a $50-\mathrm{GHz}$ channel plan.

The spectral directional division of frequency transmission is influenced by the method used for transmission stabilization; it uses acousto-optic modulators (AOMs) to produce spectral walk-off. The AOM shifts the optical frequency of the incoming optical signal $\nu_{\mathrm{O}}$ by the value given of electrical modulation signal frequency $f_{\mathrm{AOM}}$ :

$\nu_{\mathrm{T}}=\nu_{\mathrm{o}}+f_{\mathrm{AOM}}$.

The signal with a frequency $\nu_{\mathrm{T}}$ is launched into the fiber, where its frequency is subject to a shift $\nu_{\mathrm{D}}$ caused by the Doppler effect. The main contributors are thermal effects, vibrations, etc.

$\nu_{\mathrm{F}}=\nu_{\mathrm{T}}+\nu_{\mathrm{D}}=\nu_{\mathrm{o}}+f_{\mathrm{AOM}}+\nu_{\mathrm{D}}$

At the remote end, the signal with the frequency $\nu_{\mathrm{F}}$ is partially reflected and travels back to the transmitting side. Its frequency is again subject to the Doppler shift and the shift in AOM at the transmitting end:
$\nu_{\mathrm{R}}=\nu_{\mathrm{F}}+\nu_{\mathrm{D}}+f_{\mathrm{AOM}}=\nu_{\mathrm{o}}+2\left(f_{\mathrm{AOM}}+\nu_{\mathrm{D}}\right)$.

At the transmitting side, the signal is mixed with the original signal. After detection, we obtain beat frequency $f_{\mathrm{BEAT}}$ :

$f_{\mathrm{BEAT}}=\nu_{\mathrm{r}}-\nu_{\mathrm{o}}=\left(\nu_{\mathrm{o}}+2\left(f_{\mathrm{AOM}}+\nu_{\mathrm{D}}\right)=2\left(f_{\mathrm{AOM}}+\nu_{\mathrm{D}}\right)\right.$.

The $f_{\text {BEAT }}$ can be used to advantage as an error quantity to continuously modify $f_{\mathrm{AOM}}$ to compensate for the frequency shift $\nu_{\mathrm{D}}$ caused by the Doppler effect:

$f_{\mathrm{ERROR}}=-\nu_{\mathrm{D}}=-\left(f_{\mathrm{BEAT}}-2 f_{\mathrm{AOM}}\right) / 2$.

The AOM is now fed by frequency $f_{\text {AOM }}+f_{\text {ERROR }}$. At the receiving side, the signal with optical frequency $\nu_{\mathrm{T}}$ is delivered in Eq. (6):

$\nu_{\text {Ftab }}=\nu_{\mathrm{O}}+f_{\mathrm{AOM}}+f_{\mathrm{ERROR}}+\nu_{\mathrm{D}}=\nu_{\mathrm{o}}+f_{\mathrm{AOM}}=\nu_{\mathrm{T}}$.

The example of a real line Prague-Brno sharing both TF transmission is shown in Fig. 2. For more details, see Ref. 13.

Figure 3 shows a spectral measurement taken at the Prague-Brno line. We can see two time channels on the right, two frequency channels (appearing as one, due to a very low walk-off) in the middle, and amplified spontaneous emission on the left. Further in the paper, we will deeply analyze the sources of directional nonreciprocities.

\section{Nonreciprocities}

As stated earlier, we expect the reciprocity of transport delay between $\mathrm{A}$ and $\mathrm{B}$ to be $\delta=\delta \mathrm{AB}=\delta \mathrm{BA}$. However, this is not possible to achieve exactly, even during single-fiber bidirectional transmission, and we have to introduce a delay asymmetry $\Delta$ :

$\Delta=\delta \mathrm{BA}-\delta \mathrm{AB}$ 


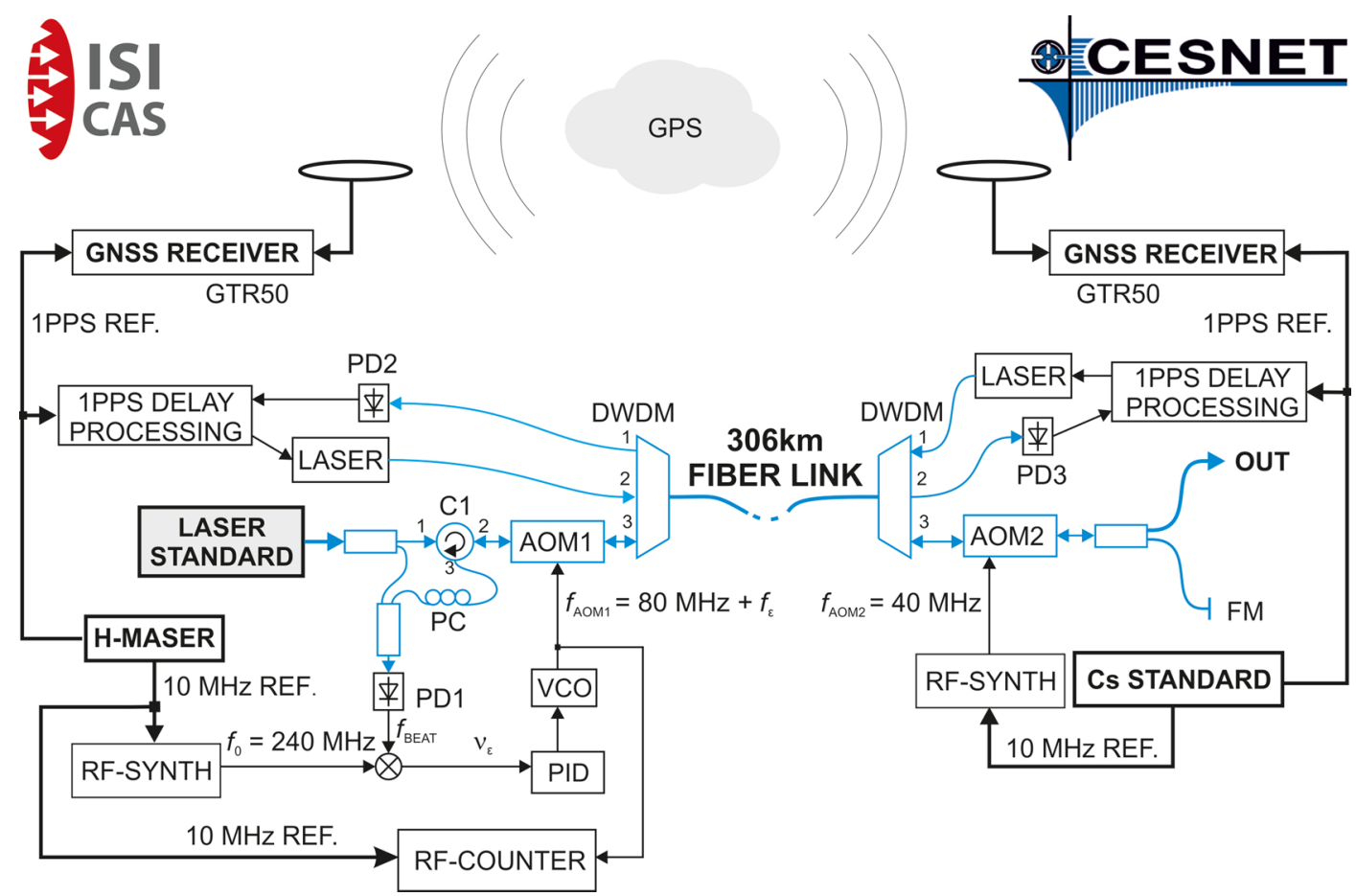

Fig. 2 Parallel transmission of precise time and stable frequency over 306-km long line.

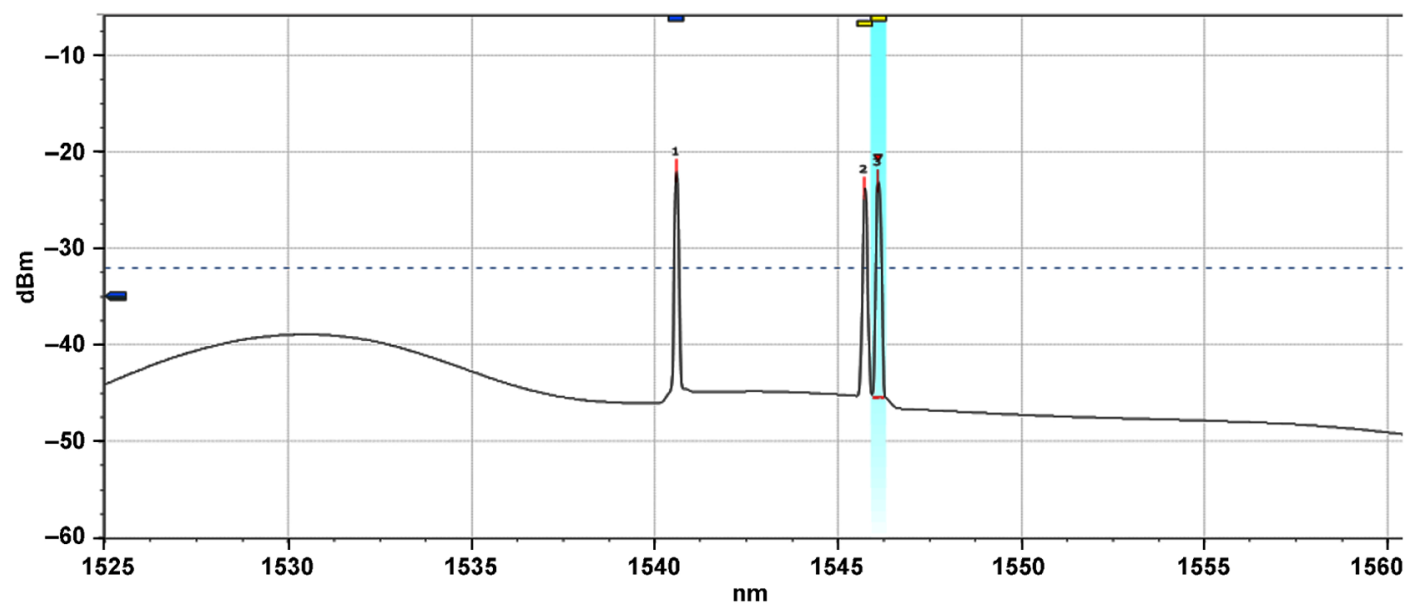

Fig. 3 Transmission spectrum-spacing example from real line.

The delay asymmetry $\Delta$ has to be measured and is a subject of a system calibration. For a picoseconds level of propagation time analysis, a precise description needs to be used.

\subsection{Calibration Issues}

There are several approaches for calibration of a time transfer system. The simple one is to compare the whole system with another independent time transfer method, e.g., one based on a Global Navigational Satellite System. Unfortunately, such a system can typically reach the accuracy of $1 \mathrm{~ns}$, according to Ref. 14, while an optical fiber-based system can be more accurate. The solution is to calibrate individual components of the optical fiber system.

Adapters: calibration of the adapters including the optical transceivers is possible by measurement of the delay between an incoming electrical signal and an outgoing optical signal at the transmitter part and, similarly, between an incoming optical and an outgoing electrical signal at the receiver part.

Optical fiber: a bidirectional optical fiber time transfer systems assume delay cancelation in both directions (with possible evaluation of residual asymmetry as discussed later) as the delay varies due to environmental influences, mainly the temperature. The single-fiber system uses the same fiber carrying signal in both directions; therefore, the physical length is the same and the delay fully cancels in case of an identical wavelength. However, two wavelengths are typically used for both directions and the difference effect is a subject of evaluation.

Amplifiers: a single path amplifier has, in principle, the same delay in both directions. Its effect on different wavelengths can be directly measured. 


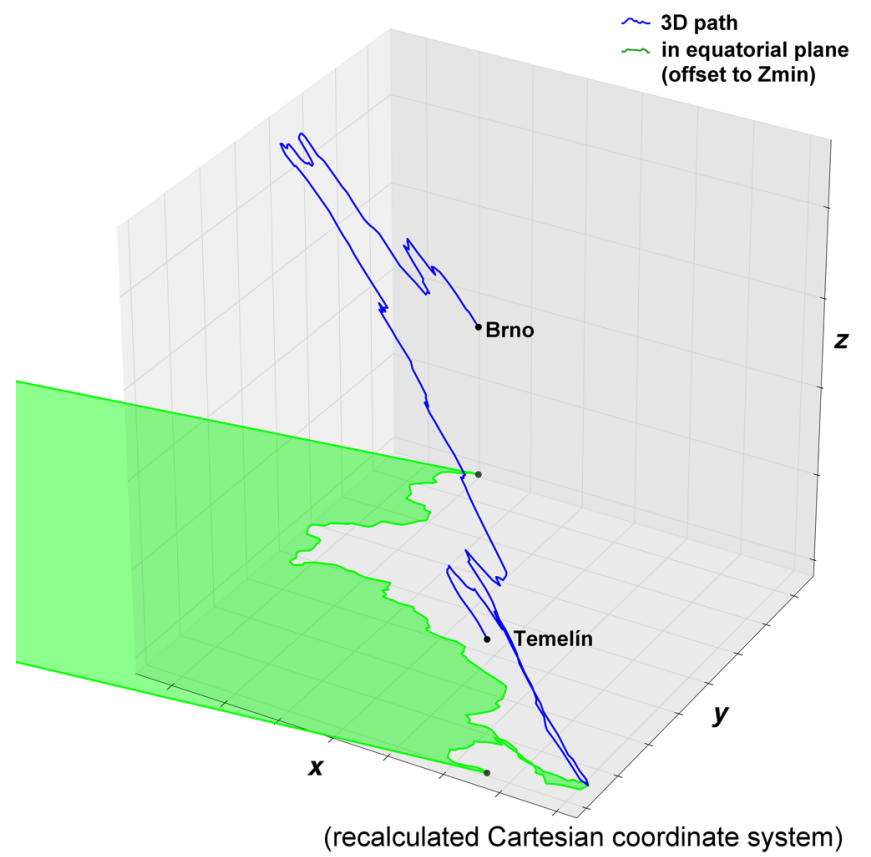

Fig. 4 Line projection to the plane perpendicular to the axis of rotation.

\subsection{Relativistic Effects}

Propagation delay is described in Eq. (8). It takes into account the relativistic effects caused by the Earth's rotation and gravitational potential. ${ }^{15}$ Only terms with a contribution higher than 1 ps on a $1000-\mathrm{km}$ long link are taken into account. $\tau$ is the propagation delay, $c$ is the speed of light in a vacuum, $L$ is the fiber path length, $n$ is the refractive index, $S$ is the Sagnac term, and $G$ is the gravitational term:

$\tau=\frac{1}{c} \int_{0}^{L} n d l \pm \frac{1}{c^{2}} \int_{0}^{L} S d l+\frac{1}{c^{3}} \int_{0}^{L} n G d l$.

The first term simply represents the influence of the refractive index, the second term describes the Sagnac effect caused by the Earth's rotation, and the third term represents the influence of the Earth's gravitational potential. The last term can be omitted for a two-way time transfer. Typical values of these terms for a 1000-km long link at the Earth's equator on the surface are $5 \mathrm{~ms}, \pm 5 \mathrm{~ns}$, and $3 \mathrm{ps}$. The higher terms can be easily omitted for picoseconds precision; ${ }^{15}$ for example, the contribution of $\mathrm{c}^{-4}$ term will be of the order $10^{-17}$ for the mentioned $1000-\mathrm{km}$ line. Equation (9) describes the contribution of the Sagnac correction to the propagation delay; the sign depends on the direction of the transfer (eastward or westward):

$|\Delta \tau|=\frac{2 \omega A}{c^{2}}$

where $\omega$ is the angular velocity of the Earth's rotation, numerically $7.2921115 \times 10^{-5} \mathrm{rad} / \mathrm{s}$. It changes in time with the Earth's tides, but, as stated in Ref. 15, the contribution to a Sagnac term Eq. (9) is only 0.3 fs for a 1000 $\mathrm{km}$ link, so it is possible to omit such influence. The last variable, $A$, is the so-called Sagnac area and corresponds to the area laying on the plane perpendicular to $\omega$ defined by the projection of the fiber path to this plane and connecting endpoints of the projected path to the rotation axis; see Fig. 4.

The exact path of a leased fiber line is not commonly available (for security reasons, etc.); typically, only some points are known, for example, see the Brno-Temelin line in Fig. 5. The basic problem is the inconsistency of a real length of fiber-its known length. The known length is represented by a set of subsequent line segments. For the real length, we have only metainformation in terms of the numerical value. We applied the method described in Ref. 14 to determine a minimal and maximal possible value of the Sagnac area A based on the given points. Furthermore, an algorithm for detection and correction of loops has been applied. Table 1 summarizes the contribution of the Sagnac effect to directional nonreciprocities for the major lines of the TF infrastructure. Corrections were determined both using a simplified model of a single line directly interconnecting endpoints and using the known fiber line points and loop detection; see Figs. 4 and 5.

\subsection{Wavelength Walk-Off}

The first term in Eq. (8) describes the influence of the refractive index, whose value is wavelength dependent for a given fiber. Thus, the used directional walk-off is a source of nonreciprocity. The difference in the propagation time of the two

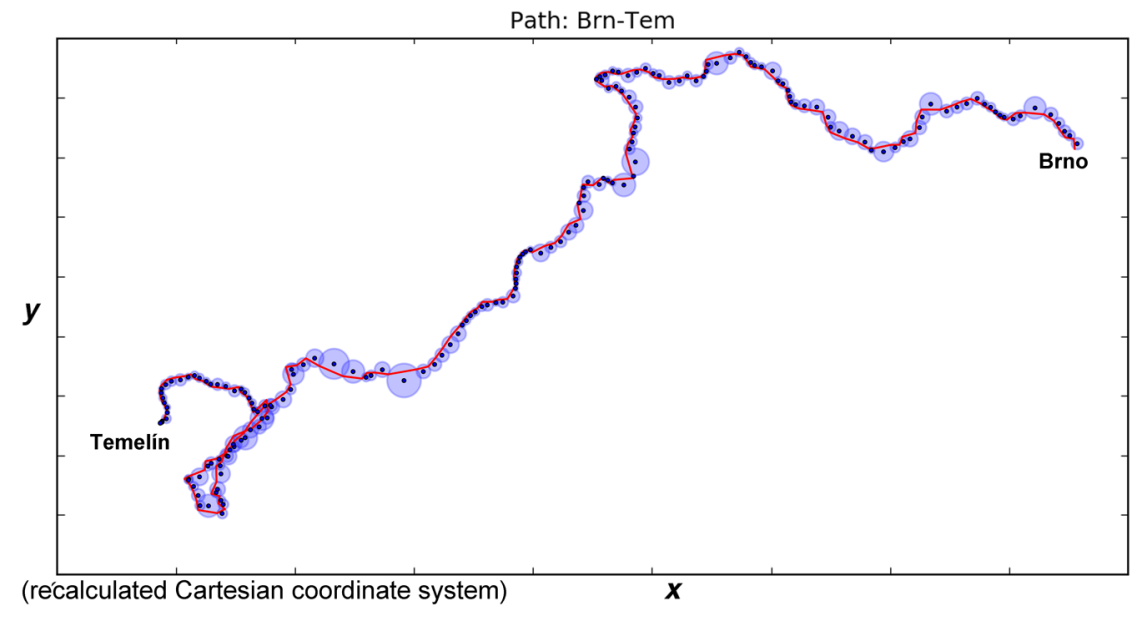

Fig. 5 Circle caps are used to determine upper and lower bounds of the path length. 
Table 1 Contribution of the Sagnac effect to directional nonreciprocities, all delays in nanoseconds.

\begin{tabular}{lcc} 
Line & $\begin{array}{c}\text { Sagnac correction }- \\
\text { direct path }\end{array}$ & $\begin{array}{c}\text { Sagnac correction - } \\
\text { segments + loops } \\
\text { detection }\end{array}$ \\
\hline Brno-Temelin & $-0.543737 \pm 0.021341$ & $-0.540961 \pm 0.000101$ \\
Rez-Brno & $0.535933 \pm 0.017503$ & $0.536158 \pm 0.000181$ \\
Chaberska, & $0.079466 \pm 0.001396$ & $0.079739 \pm 0.000004$ \\
Prague-Pecny & & \\
Prague-Brno & $0.534958 \pm 0.014714$ & $0.534447 \pm 0.000180$ \\
\hline
\end{tabular}

signals with a wavelength difference $\Delta \lambda$ is described by chromatic dispersion (CD):

$\Delta=\mathrm{CD} \cdot \Delta \lambda=(\mathrm{CD}$ coef $\cdot L+\mathrm{CD}$ pas $) \cdot \Delta \lambda$.

The total value of $\mathrm{CD}$ is given by the fiber $\mathrm{CD}$ and $\mathrm{CD}$ of the passive components, where the fiber $\mathrm{CD}$ is proportional to the fiber CD coefficient and the fiber length. A standard single-mode fiber G.652D exhibits a CD coefficient of about $17.2 \mathrm{ps} / \mathrm{nm} \cdot \mathrm{km}$ at $1550 \mathrm{~nm}$. For nonzero dispersion-shifted fibers G.655.C and G.655.E, the dispersions are 5 and $-3 \mathrm{ps} / \mathrm{nm} \cdot \mathrm{km}$, respectively.

In the presented infrastructure, the $\mathrm{CD}$ compensation is used only at one link operating over unidirectional lambdas (link Prague-Vienna). Both dispersion compensating fibers (DCF) and fiber Bragg gratings (FBG) are used as CD compensators. DCFs naturally extend the transmission path, typically adding $12 \mathrm{~km}$ to each $80-\mathrm{km}$ span. This practically decreases the optical signal-to-noise ratio due to the necessity of more optical gain. Their contribution to temperature dependence of propagation time is limited as both DCF and FBG are deployed in controlled rooms where temperature is kept reasonably stabilized. Furthermore, FBGs have an athermal design. The remaining bidirectional lines do not use CD compensation at all to avoid relatively lossy DCFs, meanwhile FBGs do not allow bidirectional operation at all. The large residual $\mathrm{CD}$ thus contributes to the uncertainty of $\Delta$ as follows. In Ref. 12, we discussed the contribution of wavelength instabilities (uncertainty of $\Delta \lambda$ ) to the uncertainty of $\Delta$, but we used only the high boundary of the CD estimation. For a precise determination of $\Delta$, we have to evaluate CD more precisely. The fiber CD coefficient is temperature dependent and real operation possesses other challenges; for example, a line might consist of fiber spans by different fiber vendors (or a vendor might be unknown) and, in the worst case, different fiber types with different CD coefficients and with unknown exact lengths of particular segments.

Also contributing to an overestimation of total CD is the fact that a TF channel contains plenty of passive devices, and a $C D$ of passive components $\mathrm{CD}_{\text {pas }}$ is provided by vendors as an upper boundary. For example, the previously mentioned line Prague-Brno contains 12 passive filters and 5 bidirectional erbium-doped fiber amplifiers (EDFAs), each containing typically 4 more passive components (directional couplers and pump couplers). Another challenge for a day-by-day operation is the fact that during any maintenance (especially unscheduled), a fiber route can be rerouted to a backup path with a very different length and sometimes a different fiber type.

The elimination of this overestimation system, based on temporary wavelength changes, has been proposed and verified. As a reference setup, two $89 \mathrm{~km}$ spans of G.652.D field deployed fiber with attenuation $22 \mathrm{~dB}$ each were interconnected into a loop and amplified by one bidirectional EDFA; for details, see Ref. 14. The smallest uncertainty of $2.2 \mathrm{ps,}$ over an averaging interval of $512 \mathrm{~s}$ in terms of time deviation, was achieved; see Fig. 6.

We deployed a periodical wavelength change over the reference line; corresponding delay changes are depicted in Fig. 7. Over a two-day measurement, we obtained an average value of $\mathrm{CD}=3017.6 \mathrm{ps} / \mathrm{nm}$ with a deviation $82.5 \mathrm{ps} / \mathrm{nm}$. As the total length is $178 \mathrm{~km}$, the resulting value is in very good correspondence with the value given by the $C D$ coefficient. Finally, the observed nonreciprocity for a $100-\mathrm{GHz}$ walk-off of transmitters was 2.424 ns, with an uncertainty of 29 ps. The observed long-term wavelength instability of transmission lasers was $\Delta \lambda=12 \mathrm{pm}$ for each transmitter laser.

In addition to the effects discussed above, a polarization mode dispersion (PMD) represents a source of uncertainty rather than directional nonreciprocity due to its stochastic

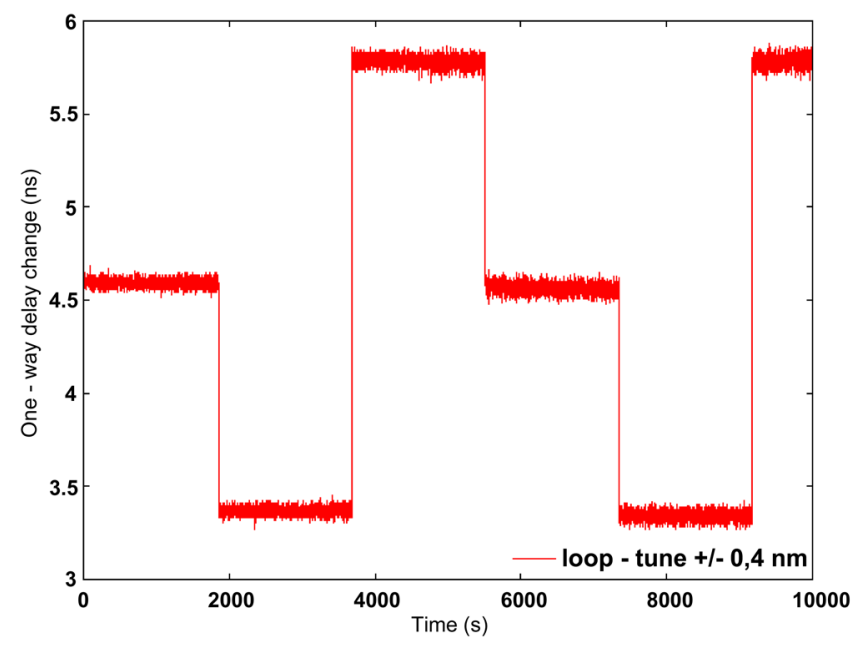

Fig. 6 Operating wavelength dependable delay, reference loop $187 \mathrm{~km}$.

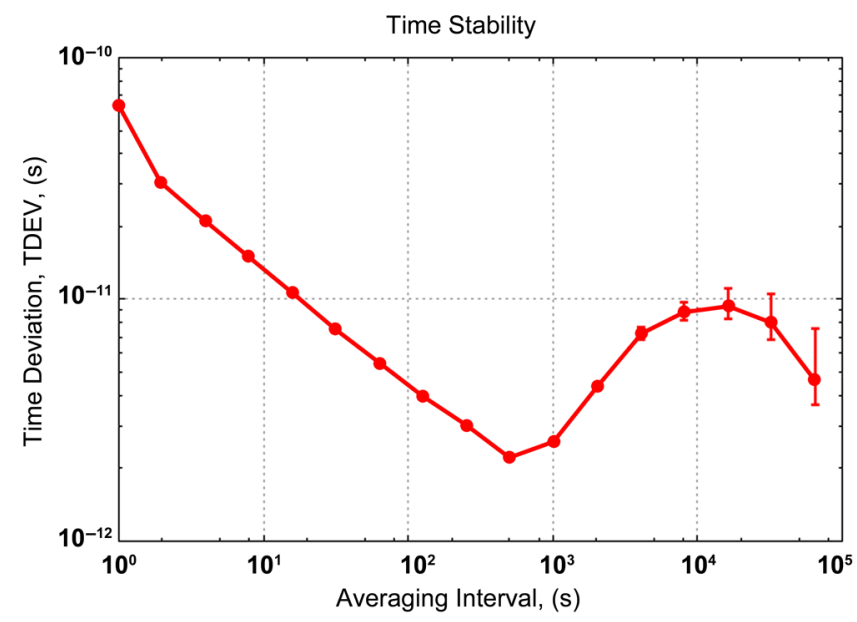

Fig. 7 Reference loop, time stability. 
nature. The used modern fibers have a PMD value limited by the PMD coefficient of $0.06 \mathrm{ps} / \mathrm{km}^{1} \frac{1}{2}$, and their maximal contribution to the uncertainty is 1.05 ps for the whole link; see Ref. 15.

We measured the PMD on some fiber links in the CESNET network and on aerial cables. Aerial cables tend to be unstable due to weather conditions and railway traffic. We found that the PMD was up to $0.4 \mathrm{ps,} \mathrm{with} \mathrm{maximum}$ variations on order of $0.1 \mathrm{ps}$. Subsequently, we may assume that the PMD for a modern optical fiber does not affect the system significantly.

We have not evaluated the real impact of the PMD of passive devices in the transmission path due to the very complicated nature of the PMD in telco equipment. Our estimate is that the total PMD for 12 passive filters is expected to be $<0.35$ ps.

\section{Conclusions}

In this paper, we briefly introduced the infrastructure for an accurate time and stable frequency transfer being gradually developed. Theoretical aspects have been verified on the real lines, which reach over $1137 \mathrm{~km}$. We also focused on the determination of bidirectional nonreciprocities caused by relativistic effects and on pilot verification of fine determination of directional nonreciprocity caused by wavelength walk-off of transmitters. By such optical TF transmissions, current satellite-based methods have been outperformed. The maximal precision of TF transmissions requires careful consideration of all contributing factors including the very small ones.

\section{Acknowledgments}

This work was supported partially by the Ministry of Education, Youth and Sport of the Czech Republic as part of the CESNET E-infrastructure project LM2015042. The issue of the phase coherent optical frequency transfer has been supported by Grant Agency of the Czech Republic (project GB14-36681G).

\section{References}

1. N. R. Newbury, "Frequency and timing distribution using optical methods," in Proc. Conf. on Lasers and Electro-Optics (CLEO '15), San Jose, California (2015).

2. S. Droste et al., "Optical frequency transfer over a single-span $1840 \mathrm{~km}$ fiber link," Phys. Rev. Lett. 111(11), 110801 (2013).

3. D. Calonico et al., "Optical frequency transfer with a $1284 \mathrm{~km}$ coherent fiber link," in Proc. European Frequency and Time Forum (EFTF '14), Neuchatel, Switzerland (2014).

4. S. C. Ebenhag et al., "Two-way coherent frequency transfer in a commercial DWDM communication network in Sweden," in Proc. Joint Conf. of the IEEE Int. Frequency Control Symp. and the European Frequency and Time Forum, Denver, Colorado, pp. 276-279 (2015).

5. H. Zhang et al., "High-precision time transfer over 2000-km fiber link," IEEE Photonics J. 7(6), 1-9 (2015).

6. O. Lopez et al., "Simultaneous remote transfer of accurate timing and optical frequency over a public fiber network," Appl. Phys. B 110(1), 3-6 (2013).

7. J. Vojtech, V. Smotlacha, and J. Radil, "All optical two-way time transfer in strongly heterogeneous networks," Proc. SPIE 9202, 92020S (2014).

8. J. Vojtech et al., "Photonic services, their enablers and applications," Proc. SPIE 8516, 85160H (2012).

9. X. Chen et al., "Simultaneously precise frequency transfer and time synchronization using feed-forward compensation technique via $120 \mathrm{~km}$ fiber link," Sci. Rep. 5, 18343 (2015).

10. V. Smotlacha, J. Vojtech, and A. Kuna, "Optical infrastructure for time and frequency transfer," in Proc. European Frequency and Time Forum and Int. Frequency Control Symp. (EFTF/IFC '13), Prague, Czech Republic, pp. 481-484 (2013).
11. J. Vojtech, V. Smotlacha, and P. Skoda, "Simultaneous transmission of accurate time in parallel with stable optical frequency in real fiber network over 612 km," in Proc. Optoelectronics Global Conf. (OGC), Shenzhen, China, pp. 1-3 (2015).

12. M. Cizek et al., "Transfer of stable optical frequency for sensory networks via 306 km optical fiber link," in Proc. European Frequency and Time Forum (EFTF '16), York, England, pp. 1-4 (2016).

13. J. Gersl, P. Delva, and P. Wolf, "Relativistic corrections for time and frequency transfer in optical fibers," Metrologia 52(4), 552-564 (2015).

14. P. Panek and A. Kuna, "Time scales comparisons using new systems and new signals," in Proc. European Navigation Conf. (ENC '13), Vienna, Austria (2013).

15. J. Vojtech et al., "Precise time transfer on the IPE - VUGKT line- a detailed analysis," in Proc. 39th Int. Conf. on Telecommunications and Signal Processing (TSP ‘16), Vienna, Austria (2016).

Josef Vojtech received his MSc degree in computer science and his PhD from the Czech Technical University in 2001 and 2008, respectively. He has more than 13 years of experience with fiber optics and has been involved in a number of international projects. He is currently the head of the Research Department of Optical Networks in CESNET. His research interests include software defined optical networking and photonic transmission. He is a member of SPIE, IEEE, and OSA.

Martin Slapak received his MSc in software engineering from Czech Technical University in Prague in 2010. His fields of interest are nature-inspired algorithms within the scope of PhD studies. He has been involved in several international projects such as GN3, GN3+, GN4, and FI-PPP XiFi.

Pavel Skoda received his MSc degree from Czech Technical University in Prague in 2008. He has 10 years of experience with fiber optics and laser communications and has been involved in a number of projects designing high-speed all-optical devices and laser systems. Besides several national projects, he participated in GN3 and GN3+ projects and joint research collaboration with Tyndall National Institute in Cork.

Jan Radil received his MSc degree and $\mathrm{PhD}$ in electrical engineering from Czech Technical University, Praha, in 1996 and 2004. He joined CESNET in 1999, where he is responsible for optical networking and development of the Czech research and educational network. He participated in EU projects SEEFIRE, Porta Optica Study, Phosphorus, GN4, XIFI, COMPLETE, and GLIF. He holds eight patents, including three US and one EU. His Hirsch index is 6.

Ondrej Havlis received his MSc degree in communications and informatics science from Brno University of Technology in 2012; currently, he is a PhD student there specializing in effective optical function in fiber photonic networks. He joined the optical networks department of CESNET in 2012. He is active in research, design, and verification of photonic services in the optical networks and development of photonic testbed. He was actively involved in the project GN3, GN3plus, and DOBI.

Michal Altmann received his MSc degree in trade and business dealing with machinery from Czech University of Life Sciences, Praha, in 2014. He joined CESNET in 2005, where he is responsible for development of the Czech research and educational network. He participated in the EU projects GN3, GN3 plus, GN4, and XIFI.

Petr Munster received his MSc degree in communication and informatics in 2010 from the Brno University of Technology and his PhD degree in 2014. Currently, he is a researcher at CESNET a.I.e. and at Brno University of Technology. His record shows more than 40 peer reviewed proceedings and journal papers in the field of fiber-optic sensing and fiber-optic telecommunications. He is a member of SPIE, IEEE, OSA, Photonics21, and the Czech and Slovak Society for Photonics.

Radek Velc received his Ing. arch. degree from the Faculty of Architecture, Czech Technical University, Prague, in 2004. He joined CESNET in 2005, where he has been working in the area of network infrastructure design and documentation within the Department of Optical Networks. He also has been engaged in the international projects COMPLETE, FI-PPP XIFI, GN4, GN3+, and GN3.

Jan Kundrat is a PhD student at the Department of Distributed and Dependable Systems at the Faculty of Mathematics and Physics, 
Charles University in Prague. He works as a researcher at CESNET, specializing in network modeling and software engineering. $\mathrm{He}$ is involved in a number of open source projects, including KDE, and has contributed to the IETF standardization process of the IMAP protocol extensions. He participated in the GN3+, FI-PPP XIFI, COMPLETE, EGI-InSpire, EGEE-III, and EGEE-II projects.

Lada Altmannova holds her MSc degree from the Czech Agriculture University in Prague, Faculty of Economy in 1983. She is interested in dark fibers lines for CESNET2 and has 15 years of extensive experience with wavelength and fiber procurements on a national level. Since 2000, she has been responsible for transformation of CESNET2 from wavelength service to dark fiber lease. She participated in the EU projects SEEFIRE, Porta Optica Study, Phosphorus, GN2, GN3, GN3+, and COMPLETE.

Rudolf Vohnout received his MSc degree in the program of transport technology and management. In 2014, he obtained his PhD in the field of applied informatics. He joined CESNET in 2011 to the Department of Optical Networking, where he mainly focuses on international projects and international cooperation in the respective field. Currently, his expertise is the optical networking procurement (PCP/PPI) and liaison of international networking services.

Tomas Horvath is a PhD student at Brno University of Technology and a researcher at CESNET. He received his MSc and BSc degrees in communications and informatics from Brno University of Technology in 2011 and 2013, respectively. His record shows more than 22 peer reviewed proceedings and journal papers. His current research interests include software-defined optical networking, passive optical networks, and sensing. He is a student member of SPIE, IEEE, and OSA.

Miloslav Hula received his MSc degree in electrical engineering from the Czech Technical University, Praha, in 2008. He joined the Research and Development Department, CESNET, Praha, in 2007, where he is responsible for optical networking and software programming for the Czech research and educational network. He has participated in the EU projects Geant2 and Phosphorus.

Vladimir Smotlacha received his MSc degree in computer science from Czech Technical University and from Charles University. He received his $\mathrm{PhD}$ degree in information science and computer engineering from the Czech Technical University. He has been with CESNET since 1996, currently in the Research and Development Department. His research interests include optical time transfer, time metrology, network time services, and communication protocols. $\mathrm{He}$ is a member of the ACM and IEEE.

Martin Cizek is a scientist at the Institute of Scientific Instruments of the Czech Academy of Sciences (ISI CAS). His research efforts concentrate mainly on electronics design (analog, digital, RF), frequency control of lasers, programming and digital signal processing, and stable optical frequency transfers over optical fiber links.

Lenka Pravdova received her master's degree in applied physics at the Palacky University, Czech Republic, where she is pursuing her $\mathrm{PhD}$. She is currently a physicist in lasers and fiber optics at the Institute of Scientific Instruments of the Czech Academy of Sciences (ISI CAS).

Simon Rerucha is a scientist at the Institute of Scientific Instruments of the Czech Academy of Sciences (ISI CAS). With the computer science background, he contributes to various research efforts that include laser interferometry methods and applications, diode laser frequency stabilization and characterization, radio-tracking equipment for zoological research, and communication and data processing infrastructure in general.

Jan Hrabina is a scientist at the Institute of Scientific Instruments of the Czech Academy of Sciences (ISI CAS). His research is oriented to the development of high-stable laser standards, investigation of optical frequency references based on absorption cells and hollow-core photonic crystal fibers filled with ultra-pure absorption gases, and multidimensional laser interferometry.

Ondrej Cip is a senior scientist at the Institute of Scientific Instruments of the Czech Academy of Sciences (ISI CAS). He is currently a head of the research group "Coherent Lasers and Interferometry" of the Department of Coherence Optics at ISI CAS. His scientific interests include frequency control of lasers, laser cooling and trapping of ions, laser spectroscopy with ultra-cold ions, and highresolution laser interferometry. 